\title{
Gated Word-Character Recurrent Language Model
}

\author{
Yasumasa Miyamoto \\ Center for Data Science \\ New York University \\ yasumasa.miyamoto@nyu.edu
}

\author{
Kyunghyun Cho \\ Courant Institute of \\ Mathematical Sciences \\ \& Centre for Data Science \\ New York University \\ kyunghyun. cho@nyu.edu
}

\begin{abstract}
We introduce a recurrent neural network language model (RNN-LM) with long shortterm memory (LSTM) units that utilizes both character-level and word-level inputs. Our model has a gate that adaptively finds the optimal mixture of the character-level and wordlevel inputs. The gate creates the final vector representation of a word by combining two distinct representations of the word. The character-level inputs are converted into vector representations of words using a bidirectional LSTM. The word-level inputs are projected into another high-dimensional space by a word lookup table. The final vector representations of words are used in the LSTM language model which predicts the next word given all the preceding words. Our model with the gating mechanism effectively utilizes the character-level inputs for rare and out-ofvocabulary words and outperforms word-level language models on several English corpora.
\end{abstract}

\section{Introduction}

Recurrent neural networks (RNNs) achieve state-ofthe-art performance on fundamental tasks of natural language processing (NLP) such as language modeling (RNN-LM) (Józefowicz et al., 2016; Zoph et al., 2016). RNN-LMs are usually based on the wordlevel information or subword-level information such as characters (Mikolov et al., 2012), and predictions are made at either word level or subword level respectively.

In word-level LMs, the probability distribution over the vocabulary conditioned on preceding words is computed at the output layer using a softmax function. ${ }^{1}$ Word-level LMs require a predefined vocabulary size since the computational complexity of a softmax function grows with respect to the vocabulary size. This closed vocabulary approach tends to ignore rare words and typos, as the words do not appear in the vocabulary are replaced with an outof-vocabulary (OOV) token. The words appearing in vocabulary are indexed and associated with highdimensional vectors. This process is done through a word lookup table.

Although this approach brings a high degree of freedom in learning expressions of words, information about morphemes such as prefix, root, and suffix is lost when the word is converted into an index. Also, word-level language models require some heuristics to differentiate between the OOV words, otherwise it assigns the exactly same vector to all the OOV words. These are the major limitations of word-level LMs.

In order to alleviate these issues, we introduce an RNN-LM that utilizes both character-level and word-level inputs. In particular, our model has a gate that adaptively choose between two distinct ways to represent each word: a word vector derived from the character-level information and a word vector stored in the word lookup table. This gate is trained to make this decision based on the input word.

According to the experiments, our model with the gate outperforms other models on the Penn Treebank (PTB), BBC, and IMDB Movie Review datasets. Also, the trained gating values show that the gating mechanism effectively utilizes the character-level

\footnotetext{
${ }^{1}$ softmax function is defined as $f\left(x_{i}\right)=\frac{\exp x_{i}}{\sum_{k} \exp x_{k}}$.
} 
information when it encounters rare words.

Related Work Character-level language models that make word-level prediction have recently been proposed. Ling et al. (2015a) introduce the compositional character-to-word $(\mathrm{C} 2 \mathrm{~W})$ model that takes as input character-level representation of a word and generates vector representation of the word using a bidirectional LSTM (Graves and Schmidhuber, 2005). Kim et al. (2015) propose a convolutional neural network (CNN) based character-level language model and achieve the state-of-the-art perplexity on the PTB dataset with a significantly fewer parameters.

Moreover, word-character hybrid models have been studied on different NLP tasks. Kang et al. (2011) apply a word-character hybrid language model on Chinese using a neural network language model (Bengio et al., 2003). Santos and Zadrozny (2014) produce high performance part-of-speech taggers using a deep neural network that learns character-level representation of words and associates them with usual word representations. Bojanowski et al. (2015) investigate RNN models that predict characters based on the character and word level inputs. Luong and Manning (2016) present word-character hybrid neural machine translation systems that consult the character-level information for rare words.

\section{Model Description}

The model architecture of the proposed wordcharacter hybrid language model is shown in Fig. 1.

Word Embedding At each time step $t$, both the word lookup table and a bidirectional LSTM take the same word $w_{t}$ as an input. The word-level input is projected into a high-dimensional space by a word lookup table $\mathbf{E} \in \mathbb{R}^{|V| \times d}$, where $|V|$ is the vocabulary size and $d$ is the dimension of a word vector:

$$
\mathbf{x}_{w_{t}}^{\text {word }}=\mathbf{E}^{\top} \mathbf{w}_{w_{t}}
$$

where $\mathbf{w}_{w_{t}} \in \mathbb{R}^{|V|}$ is a one-hot vector whose $i$-th element is 1 , and other elements are 0 . The characterlevel input is converted into a word vector by using a bidirectional LSTM. The last hidden states of forward and reverse recurrent networks are linearly

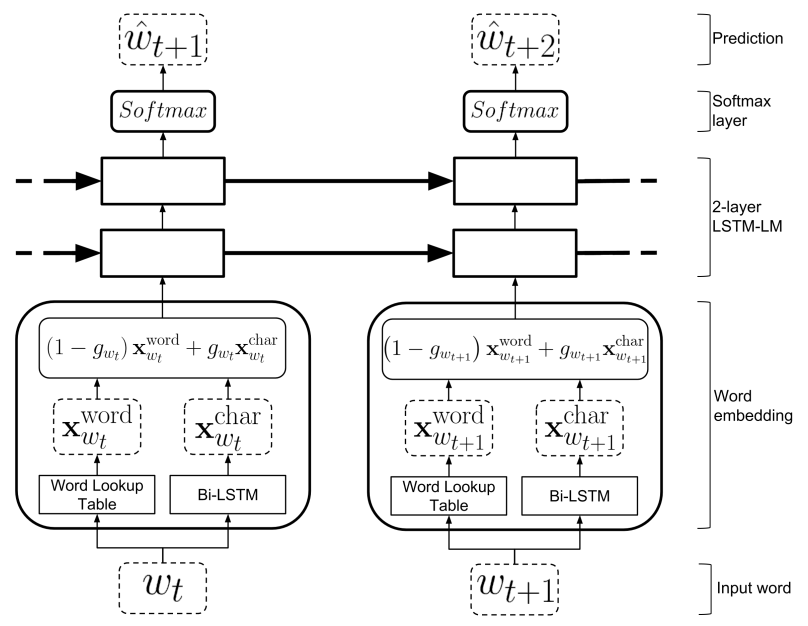

Figure 1: The model architecture of the gated word-character recurrent language model. $w_{t}$ is an input word at $t . \mathbf{x}_{w_{t}}^{\text {word }}$ is a word vector stored in the word lookup table. $\mathbf{x}_{w_{t}}^{\text {char }}$ is a word vector derived from the character-level input. $g_{w_{t}}$ is a gating value of a word $w_{t}$. $\hat{w}_{t+1}$ is a prediction made at $t$.

combined:

$$
\mathbf{x}_{w_{t}}^{\text {char }}=\mathbf{W}^{f} \mathbf{h}_{w_{t}}^{f}+\mathbf{W}^{r} \mathbf{h}_{w_{t}}^{r}+\mathbf{b},
$$

where $\mathbf{h}_{w_{t}}^{f}, \mathbf{h}_{w_{t}}^{r} \in \mathbb{R}^{d}$ are the last states of the forward and the reverse LSTM respectively. $\mathbf{W}^{f}, \mathbf{W}^{r} \in \mathbb{R}^{d \times d}$ and $\mathbf{b} \in \mathbb{R}^{d}$ are trainable parameters, and $\mathbf{x}_{w_{t}}^{\text {char }} \in \mathbb{R}^{d}$ is the vector representation of the word $w_{t}$ using a character input. The generated vectors $\mathbf{x}_{w_{t}}^{\text {word }}$ and $\mathbf{x}_{w_{t}}^{\text {char }}$ are mixed by a gate $g_{w_{t}}$ as

$$
\begin{aligned}
& g_{w_{t}}=\sigma\left(\mathbf{v}_{g}^{\top} \mathbf{x}_{w_{t}}^{\text {word }}+b_{g}\right) \\
& \mathbf{x}_{w_{t}}=\left(1-g_{w_{t}}\right) \mathbf{x}_{w_{t}}^{\text {word }}+g_{w_{t}} \mathbf{x}_{w_{t}}^{\text {char }}
\end{aligned}
$$

where $\mathbf{v}_{g} \in \mathbb{R}^{d}$ is a weight vector, $b_{g} \in \mathbb{R}$ is a bias scalar, $\sigma(\cdot)$ is a sigmoid function. This gate value is independent of a time step. Even if a word appears in different contexts, the same gate value is applied. Hashimoto and Tsuruoka (2016) apply a very similar approach to compositional and noncompositional phrase embeddings and achieve stateof-the-art results on compositionality detection and verb disambiguation tasks.

Language Modeling The output vector $\mathbf{x}_{w_{t}}$ is used as an input to a LSTM language model. Since the word embedding part is independent from the language modeling part, our model retains the flexibility to change the architecture of the language modeling part. We use the architecture similar to the nonregularized LSTM model by Zaremba et al. (2014). 


\begin{tabular}{l||cc|cc|cc}
\hline \multicolumn{1}{c|}{ Model } & \multicolumn{2}{c|}{ PTB } & \multicolumn{2}{c|}{ BBC } & \multicolumn{2}{c}{ IMDB } \\
\cline { 2 - 7 } \multicolumn{1}{c|}{ Validation } & Test & Validation & Test & Validation & Test \\
\hline Gated Word \& Char, adaptive & 117.49 & 113.87 & $\mathbf{7 8 . 5 6}$ & $\mathbf{8 7 . 1 6}$ & 71.99 & 72.29 \\
Gated Word \& Char, adaptive (Pre-train) & 117.03 & 112.90 & 80.37 & 87.51 & 71.16 & 71.49 \\
\hline Gated Word \& Char, $g=0.25$ & 119.45 & 115.55 & 79.67 & 88.04 & 71.81 & 72.14 \\
Gated Word \& Char, $g=0.25$ (Pre-train) & $\mathbf{1 1 7 . 0 1}$ & $\mathbf{1 1 3 . 5 2}$ & 80.07 & 87.99 & $\mathbf{7 0 . 6 0}$ & $\mathbf{7 0 . 8 7}$ \\
Gated Word \& Char, $g=0.5$ & 126.01 & 121.99 & 89.27 & 94.91 & 106.78 & 107.33 \\
Gated Word \& Char, $g=0.5$ (Pre-train) & 117.54 & 113.03 & 82.09 & 88.61 & 109.69 & 110.28 \\
Gated Word \& Char, $g=0.75$ & 135.58 & 135.00 & 105.54 & 111.47 & 115.58 & 116.02 \\
Gated Word \& Char, $g=0.75$ (Pre-train) & 179.69 & 172.85 & 132.96 & 136.01 & 106.31 & 106.86 \\
\hline Word Only & 118.03 & 115.65 & 84.47 & 90.90 & 72.42 & 72.75 \\
Character Only & 132.45 & 126.80 & 88.03 & 97.71 & 98.10 & 98.59 \\
Word \& Character & 125.05 & 121.09 & 88.77 & 95.44 & 77.94 & 78.29 \\
Word \& Character (Pre-train) & 122.31 & 118.85 & 84.27 & 91.24 & 80.60 & 81.01 \\
\hline Non-regularized LSTM (Zaremba, 2014) & 120.7 & 114.5 & - & - & - & - \\
\hline
\end{tabular}

Table 1: Validation and test perplexities on Penn Treebank (PTB), BBC, IMDB Movie Reviews datasets.

One step of LSTM computation corresponds to

$$
\begin{aligned}
\mathbf{f}_{t} & =\sigma\left(\mathbf{W}_{f} \mathbf{x}_{w_{t}}+\mathbf{U}_{f} \mathbf{h}_{t-1}+\mathbf{b}_{f}\right) \\
\mathbf{i}_{t} & =\sigma\left(\mathbf{W}_{i} \mathbf{x}_{w_{t}}+\mathbf{U}_{i} \mathbf{h}_{t-1}+\mathbf{b}_{i}\right) \\
\tilde{\mathbf{c}}_{t} & =\tanh \left(\mathbf{W}_{\tilde{c}} \mathbf{x}_{w_{t}}+\mathbf{U}_{\tilde{c}} \mathbf{h}_{t-1}+\mathbf{b}_{\tilde{c}}\right) \\
\mathbf{o}_{t} & =\sigma\left(\mathbf{W}_{o} \mathbf{x}_{w_{t}}+\mathbf{U}_{o} \mathbf{h}_{t-1}+\mathbf{b}_{o}\right) \\
\mathbf{c}_{t} & =\mathbf{f}_{t} \odot \mathbf{c}_{t-1}+\mathbf{i}_{t} \odot \tilde{\mathbf{c}}_{t} \\
\mathbf{h}_{t} & =\mathbf{o}_{t} \odot \tanh \left(\mathbf{c}_{t}\right),
\end{aligned}
$$

where $\mathbf{W}_{s}, \mathbf{U}_{s} \in \mathbb{R}^{d \times d}$ and $\mathbf{b}_{s} \in \mathbb{R}^{d}$ for $s \in$ $\{f, i, \tilde{c}, o\}$ are parameters of LSTM cells. $\sigma(\cdot)$ is an element-wise sigmoid function, $\tanh (\cdot)$ is an element-wise hyperbolic tangent function, and $\odot$ is an element-wise multiplication.

The hidden state $\mathbf{h}_{t}$ is affine-transformed followed by a softmax function:

$$
\operatorname{Pr}\left(w_{t+1}=k \mid w_{<t+1}\right)=\frac{\exp \left(\mathbf{v}_{k}^{\top} \mathbf{h}_{t}+b_{k}\right)}{\sum_{k^{\prime}} \exp \left(\mathbf{v}_{k^{\prime}}^{\top} \mathbf{h}_{t}+b_{k^{\prime}}\right)},
$$

where $\mathbf{v}_{k}$ is the $k$-th column of a parameter matrix $\mathbf{V} \in \mathbb{R}^{d \times|V|}$ and $b_{k}$ is the $k$-th element of a bias vector $\mathbf{b} \in \mathbb{R}^{d}$. In the training phase, we minimizes the negative log-likelihood with stochastic gradient descent.

\section{Experimental Settings}

We test five different model architectures on the three English corpora. Each model has a unique word embedding method, but all models share the same LSTM language modeling architecture, that has 2 LSTM layers with 200 hidden units, $d=200$. Except for the character only model, weights in the language modeling part are initialized with uniform random variables between -0.1 and 0.1 . Weights of a bidirectional LSTM in the word embedding part are initialized with Xavier initialization (Glorot and Bengio, 2010). All biases are initialized to zero.

Stochastic gradient decent (SGD) with mini-batch size of 32 is used to train the models. In the first $k$ epochs, the learning rate is 1 . After the $k$-th epoch, the learning rate is divided by $l$ each epoch. $k$ manages learning rate decay schedule, and $l$ controls speed of decay. $k$ and $l$ are tuned for each model based on the validation dataset.

As the standard metric for language modeling, perplexity (PPL) is used to evaluate the model performance. Perplexity over the test set is computed as PPL $=\exp \left(-\frac{1}{N} \sum_{i=1}^{N} \log p_{\left(w_{i} \mid w_{<i}\right)}\right)$, where $N$ is the number of words in the test set, and $p_{\left(w_{i} \mid w_{<i}\right)}$ is the conditional probability of a word $w_{i}$ given all the preceding words in a sentence. We use Theano (2016) to implement all the models. The code for the models is available from https://gi thub.com/ nyu-dl/gated_word_char_rlm.

\subsection{Model Variations}

Word Only (baseline) This is a traditional wordlevel language model and is a baseline model for our experiments.

Character Only This is a language model where each input word is represented as a character se- 


\begin{tabular}{ll|ccc}
\hline & & Train & Validation & test \\
\hline \multirow{2}{*}{ PTB } & \# Sentences & $42 \mathrm{k}$ & $3 \mathrm{k}$ & $4 \mathrm{k}$ \\
& \# Word & $888 \mathrm{k}$ & $70 \mathrm{k}$ & $79 \mathrm{k}$ \\
\hline \multirow{2}{*}{ BBC } & \# Sentences & $37 \mathrm{k}$ & $2 \mathrm{k}$ & $2 \mathrm{k}$ \\
& \# Word & $890 \mathrm{k}$ & $49 \mathrm{k}$ & $53 \mathrm{k}$ \\
\hline \multirow{2}{*}{ IMDB } & \# Sentences & $930 \mathrm{k}$ & $153 \mathrm{k}$ & $152 \mathrm{k}$ \\
& \# Word & $21 \mathrm{M}$ & $3 \mathrm{M}$ & $3 \mathrm{M}$ \\
\hline
\end{tabular}

Table 2: The size of each dataset.

quence similar to the $\mathrm{C} 2 \mathrm{~W}$ model in (Ling et al., 2015a). The bidirectional LSTMs have 200 hidden units, and their weights are initialized with Xavier initialization. In addition, the weights of the forget, input, and output gates are scaled by a factor of 4 . The weights in the LSTM language model are also initialized with Xavier initialization. All biases are initialized to zero. A learning rate is fixed at 0.2 .

Word \& Character This model simply concatenates the vector representations of a word constructed from the character input $\mathbf{x}_{w_{t}}^{\text {char }}$ and the word input $\mathbf{x}_{w_{t}}^{\text {word }}$ to get the final representation of a word $\mathbf{x}_{w_{t}}$, i.e.,

$$
\mathbf{x}_{w_{t}}=\left[\mathbf{x}_{w_{t}}^{\mathrm{char}} ; \mathbf{x}_{w_{t}}^{\text {word }}\right] .
$$

Before being concatenated, the dimensions of $\mathbf{x}_{w_{t}}^{\text {char }}$ and $\mathbf{x}_{w_{t}}^{\text {word }}$ are reduced by half to keep the size of $\mathbf{x}_{w_{t}}$ comparably to other models.

Gated Word \& Character, Fixed Value This model uses a globally constant gating value to combine vector representations of a word constructed from the character input $\mathbf{x}_{w_{t}}^{\text {char }}$ and the word input $\mathbf{x}_{w_{t}}^{\text {word }}$ as

$$
\mathbf{x}_{w_{t}}=(1-g) \mathbf{x}_{w_{t}}^{\text {word }}+g \mathbf{x}_{w_{t}}^{\text {char }},
$$

where $g$ is some number between 0 and 1 . We choose $g=\{0.25,0.5,0.75\}$.

Gated Word \& Character, Adaptive This model uses adaptive gating values to combine vector representations of a word constructed from the character input $\mathbf{x}_{w_{t}}^{\text {char }}$ and the word input $\mathbf{x}_{w_{t}}^{\text {word }}$ as the Eq (3).

\subsection{Datasets}

Penn Treebank We use the Penn Treebank Corpus (Marcus et al., 1993) preprocessed by Mikolov et al. (2010). We use 10k most frequent words and 51 characters. In the training phase, we use only sentences with less than 50 words.
BBC We use the BBC corpus prepared by Greene \& Cunningham (2006). We use 10k most frequent words and 62 characters. In the training phase, we use sentences with less than 50 words.

IMDB Movie Reviews We use the IMDB Move Review Corpus prepared by Maas et al. (2011). We use $30 \mathrm{k}$ most frequent words and 74 characters. In the training phase, we use sentences with less than 50 words. In the validation and test phases, we use sentences with less than 500 characters.

\subsection{Pre-training}

For the word-character hybrid models, we applied a pre-training procedure to encourage the model to use both representations. The entire model is trained only using the word-level input for the first $m$ epochs and only using the character-level input in the next $m$ epochs. In the first $m$ epochs, a learning rate is fixed at 1 , and a smaller learning rate 0.1 is used in the next $m$ epochs. After the $2 m$-th epoch, both the character-level and the word-level inputs are used. We use $m=2$ for PTB and BBC, $m=1$ for IMDB.

Lample et al. (2016) report that a pre-trained word lookup table improves performance of their word \& character hybrid model on named entity recognition (NER). In their method, word embeddings are first trained using skip-n-gram (Ling et al., 2015b), and then the word embeddings are finetuned in the main training phase.

\section{Results and Discussion}

\subsection{Perplexity}

Table 1 compares the models on each dataset. On the PTB and IMDB Movie Review dataset, the gated word \& character model with a fixed gating value, $g_{\text {const }}=0.25$, and pre-training achieves the lowest perplexity . On the BBC datasets, the gated word $\&$ character model without pre-training achieves the lowest perplexity.

Even though the model with fixed gating value performs well, choosing the gating value is not clear and might depend on characteristics of datasets such as size. The model with adaptive gating values does not require tuning it and achieves similar perplexity. 


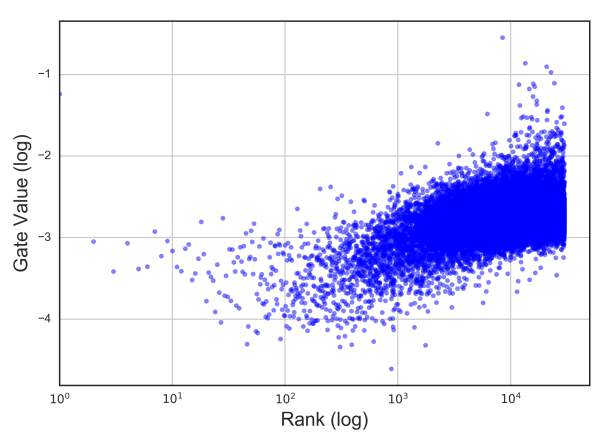

(a) Gated word \& character.

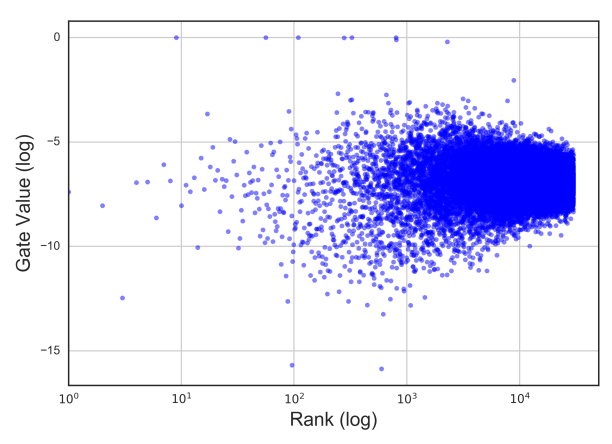

(b) Gated word \& character with pre-taining.

Figure 2: A log-log plot of frequency ranks and gating values trained in the gated word \& character models with/without pretraining.

\subsection{Values of Word-Character Gate}

The BBC and IMDB datasets retain out-ofvocabulary $(\mathrm{OOV})$ words while the OOV words have been replaced by <unk> in the Penn Treebank dataset. On the BBC and IMDB datasets, our model assigns a significantly high gating value on the unknown word token UNK compared to the other words.

We observe that pre-training results the different distributions of gating values. As can be seen in Fig. 2 (a), the gating value trained in the gated word $\&$ character model without pre-training is in general higher for less frequent words, implying that the recurrent language model has learned to exploit the spelling of a word when its word vector could not have been estimated properly. Fig. 2 (b) shows that the gating value trained in the gated word $\&$ character model with pre-training is less correlated with the frequency ranks than the one without pre-training. The pre-training step initializes a word lookup table using the training corpus and includes its information into the initial values. We hypothesize that the recurrent language model tends to be word-inputoriented if the informativeness of word inputs and character inputs are not balanced especially in the early stage of training.

Although the recurrent language model with or without pre-training derives different gating values, the results are still similar. We conjecture that the flexibility of modulating between word-level and character-level representations resulted in a better language model in multiple ways.

Overall, the gating values are small. However, this does not mean the model does not utilize the character-level inputs. We observed that the word vectors constructed from the character-level inputs usually have a larger L2 norm than the word vectors constructed from the word-level inputs do. For instance, the mean values of L2 norm of the 1000 most frequent words in the IMDB training set are 52.77 and 6.27 respectively. The small gate values compensate for this difference.

\section{Conclusion}

We introduced a recurrent neural network language model with LSTM units and a word-character gate. Our model was empirically found to utilize the character-level input especially when the model encounters rare words. The experimental results suggest the gate can be efficiently trained so that the model can find a good balance between the wordlevel and character-level inputs.

\section{Acknowledgments}

This work is done as a part of the course DSGA 1010-001 Independent Study in Data Science at the Center for Data Science, New York University. KC thanks the support by Facebook, Google (Google Faculty Award 2016) and NVidia (GPU Center of Excellence 2015-2016). YM thanks Kentaro Hanaki, Israel Malkin, and Tian Wang for their helpful feedback. KC and YM thanks the anonymous reviewers for their insightful comments and suggestions. 


\section{References}

[Bengio et al.2003] Yoshua Bengio, Réjean Ducharme, Pascal Vincent, and Christian Janvin. 2003. A neural probabilistic language model. Journal of Machine Learning Research, 3:1137-1155.

[Bojanowski et al.2015] Piotr Bojanowski, Armand Joulin, and Tomas Mikolov. 2015. Alternative structures for character-level rnns. CoRR, abs/1511.06303.

[dos Santos and Zadrozny2014] Cícero Nogueira dos Santos and Bianca Zadrozny. 2014. Learning character-level representations for part-of-speech tagging. In Proceedings of the 31th International Conference on Machine Learning, ICML 2014, Beijing, China, 21-26 June 2014, pages 1818-1826.

[Glorot and Bengio2010] Xavier Glorot and Yoshua Bengio. 2010. Understanding the difficulty of training deep feedforward neural networks. In Proceedings of the Thirteenth International Conference on Artificial Intelligence and Statistics, AISTATS 2010, Chia Laguna Resort, Sardinia, Italy, May 13-15, 2010, pages 249-256.

[Graves and Schmidhuber2005] Alex Graves and Jürgen Schmidhuber. 2005. Framewise phoneme classification with bidirectional LSTM and other neural network architectures. Neural Networks, 18(5-6):602-610.

[Greene and Cunningham2006] Derek Greene and Padraig Cunningham. 2006. Practical solutions to the problem of diagonal dominance in kernel document clustering. In Machine Learning, Proceedings of the Twenty-Third International Conference (ICML 2006), Pittsburgh, Pennsylvania, USA, June 25-29, 2006, pages 377-384.

[Hashimoto and Tsuruoka2016] Kazuma Hashimoto and Yoshimasa Tsuruoka. 2016. Adaptive joint learning of compositional and non-compositional phrase embeddings. CoRR, abs/1603.06067.

[Józefowicz et al.2016] Rafal Józefowicz, Oriol Vinyals, Mike Schuster, Noam Shazeer, and Yonghui Wu. 2016. Exploring the limits of language modeling. CoRR, abs/1602.02410.

[Kang et al.2011] Moonyoung Kang, Tim Ng, and Long Nguyen. 2011. Mandarin word-character hybridinput neural network language model. In INTERSPEECH 2011, 12th Annual Conference of the International Speech Communication Association, Florence, Italy, August 27-31, 2011, pages 625-628.

[Kim et al.2015] Yoon Kim, Yacine Jernite, David Sontag, and Alexander M. Rush. 2015. Character-aware neural language models. CoRR, abs/1508.06615.

[Lample et al.2016] Guillaume Lample, Miguel Ballesteros, Sandeep Subramanian, Kazuya Kawakami, and Chris Dyer. 2016. Neural architectures for named entity recognition. CoRR, abs/1603.01360.
[Ling et al.2015a] Wang Ling, Tiago Luís, Luís Marujo, Rámon Fernandez Astudillo, Silvio Amir, Chris Dyer, Alan W Black, and Isabel Trancoso. 2015a. Finding function in form: Compositional character models for open vocabulary word representation. EMNLP.

[Ling et al.2015b] Wang Ling, Yulia Tsvetkov, Silvio Amir, Ramon Fermandez, Chris Dyer, Alan W. Black, Isabel Trancoso, and Chu-Cheng Lin. 2015b. Not all contexts are created equal: Better word representations with variable attention. In Proceedings of the 2015 Conference on Empirical Methods in Natural Language Processing, EMNLP 2015, Lisbon, Portugal, September 17-21, 2015, pages 1367-1372.

[Luong and Manning2016] Minh-Thang Luong and Christopher D. Manning. 2016. Achieving open vocabulary neural machine translation with hybrid word-character models. CoRR, abs/1604.00788.

[Maas et al.2011] Andrew L. Maas, Raymond E. Daly, Peter T. Pham, Dan Huang, Andrew Y. Ng, and Christopher Potts. 2011. Learning word vectors for sentiment analysis. In The 49th Annual Meeting of the Association for Computational Linguistics: $\mathrm{Hu}$ man Language Technologies, Proceedings of the Conference, 19-24 June, 2011, Portland, Oregon, USA, pages 142-150.

[Marcus et al.1993] Mitchell P. Marcus, Beatrice Santorini, and Mary Ann Marcinkiewicz. 1993. Building a large annotated corpus of english: The penn treebank. Computational Linguistics, 19(2):313-330.

[Mikolov et al.2010] Tomas Mikolov, Martin Karafiát, Lukás Burget, Jan Cernocký, and Sanjeev Khudanpur. 2010. Recurrent neural network based language model. In INTERSPEECH 2010, 11th Annual Conference of the International Speech Communication Association, Makuhari, Chiba, Japan, September 26-30, 2010, pages 1045-1048.

[Mikolov et al.2012] Tomas Mikolov, Ilya Sutskever, Anoop Deoras, Hai-Son Le, and Stefan Kombrink. 2012. Subword language modeling with neural networks.

[Theano Development Team2016] Theano Development Team. 2016. Theano: A Python framework for fast computation of mathematical expressions. arXiv $e$ prints, abs/1605.02688, May.

[Zaremba et al.2014] Wojciech Zaremba, Ilya Sutskever, and Oriol Vinyals. 2014. Recurrent neural network regularization. CoRR, abs/1409.2329.

[Zoph et al.2016] Barret Zoph, Ashish Vaswani, Jonathan May, and Kevin Knight. 2016. Simple, fast noise-contrastive estimation for large rnn vocabularies. NAACL. 